\title{
A new Monte Carlo mobile node localization algorithm based on Newton interpolation
}

\author{
Jian-Yin Lu $^{1 *}$ and Chao Wang ${ }^{2}$
}

\begin{abstract}
There are some deficiencies in the Monte Carlo localization algorithm based on rangefinder, which like location probability distribution of the $k$ moment in the prediction phase only related to the localization of the $k-1$ moment and the maximum and minimum velocity. And the influences of the motion condition on the movement of the mobile node at $k$ moment are also not considered before the $k-1$ moment. What is more is the process of selecting the effective particles is slow in the algorithm. Considering the situations above, this paper presented a Monte Carlo mobile node localization algorithm based on Newton interpolation, which uses the inheritedness of Newton interpolation, inheriting the historical trajectory prediction mechanism of the moving node to estimate the current moment's movement speed and movement direction of the moving node, and optimized the moving node motion model, and used particle filter that is optimized by weight of importance to prevent particle collection depletion. The inference and simulation results show that the algorithm has improved the accuracy of the forecast using Newton interpolation. And this algorithm has effectively avoided the degradation of particles and improved the localization accuracy.
\end{abstract}

Keywords: Newton interpolation, Monte Carlo, Localization algorithm

\section{Introduction}

In recent years, with the rapid development of micro electro mechanical systems and wireless communication technology, wireless sensor network has been applying in wisdom city, wisdom tourist, intelligent household, wisdom pension, and many other areas as a kind of brand-new information acquisition and data processing technology. The application of wireless sensor network depends all on the position information of nodes, and mobile node localization's technical difficulty compared with the stationary node localization is more complex, which is also an important technology that is based on location service. Therefore, how to design the location tracking algorithm for wireless sensor mobile network environment with the movement information of the node has been becoming a hot issue for many researchers, and

\footnotetext{
* Correspondence: jianyinlu@163.com

${ }^{1}$ College of Information Engineering, Chao Hu University, Hefei, Anhui, China Full list of author information is available at the end of the article
}

this research is quiet important for practical significance and application value.

$\mathrm{Hu}$ and Evans first proposed the algorithm that is based on Sequential Monte Carlo Localization algorithm [1, 2] and has achieved good results on location of mobile sensor network node. Frank et al. first applied Monte Carlo localization (MCL) $[3,4]$ to robot location, and the core is based on Bayes filter location estimation to estimate the position distribution of mobile robot in state space using weighted particle set, but Bayes filtering made the previously measured data and current measurements relatively independent [5]. Aline Baggio et al. have proposed Monte Carlo Localization Boxed algorithm [6] which is an improvement of MCL algorithm that take overlapping areas within communication range as the sampling range by building beacon box and check box to optimize the sampling area and improve the positioning performance [7]. But the sampling effect of the MCB algorithm is not good when the observed data distributed in the anchor node box rarely. However, sampling success rate is one of the main indexes to measure the positioning performance of
Springer Open

(c) The Author(s). 2018 Open Access This article is distributed under the terms of the Creative Commons Attribution 4.0 International License (http://creativecommons.org/licenses/by/4.0/), which permits unrestricted use, distribution, and reproduction in any medium, provided you give appropriate credit to the original author(s) and the source, provide a link to the Creative Commons license, and indicate if changes were made. 
mobile nodes. The localization algorithm based on received signal strength indication, RSSI [8], has the advantages of low cost and easy implementation and is widely used in the actual localization system with low accuracy requirement. In nowadays, most radio frequency chips themselves have RSSI information that receives radio frequency data so that requires no additional ranging hardware. So, RSSI measurements are cheap and easy to implement, but due to the multi-diameter, interference, occlusion, and other factors, the positioning algorithm of single RSSI is not high enough to meet the requirements [9] of precise locating. Now, many scholars and researchers have taken advantages of the full study of RSSI, which means using ranging information to optimize the non-ranging location to propose a Monte Carlo localization algorithm based on rangefinder [10]. This algorithm used the distance information obtained by statistical model as the observation value in the filter stage of Monte Carlo algorithm, which significantly improves the location accuracy of MCL algorithm. In the prediction phase of the algorithm, the position probability distribution of moment $k$ is only related to the location of the $k-1$ moment and the maximum or minimum speed, rather than accounting the influence of motion velocity and movement direction on the current mobile nodes before the $k-1$ moment, and the process of selecting effective particles is slow in the posterior distribution phase of the algorithm.

In this paper, a mobile node 1 localization algorithm, received signal strength indication improvement Monte Carlo localization, RSSI-IMCL, is proposed, and in this algorithm, Newton interpolation method is used to predict the location of the estimated nodes based on the historical trajectory of the node and optimize the speed and direction of the node movement. In addition, this paper optimized the particle filter algorithm about particle importance weights by introducing a weight impact factors, which made more particles being copied in the resampling process, effectively avoid the particle degeneration, so as to improve the localization accuracy of nodes.

\section{RSSI-IMCL localization algorithm model}

\subsection{Ranging statistics model}

In wireless communication system, the level of the receiving signal reflects the distance between the sending node and receiving nodes, and RSSI value can be used to calculate the distance between the receiving node and transmitting node by the radio frequency transmission loss model. The RSSI localization algorithm is the location information that uses the distance of its own computation and the known sending node coordinates to calculate its Euclidean geometry relation, and then gets the receiving node. However, the RSSI range has a large error, mainly from the radio frequency transmission loss model, and because the loss model of radio frequency propagation is very complicated in practical application, the simple mathematical model does not reflect the change of different factors like multipath, interference, and occlusion in each actual environment. In practical application, the RSSI values that obtained by measure are generally consistent with a statistical rule based on radio frequency transmission loss model, which is called RSSI statistical model. In general, a small amount of RSSI value is not the same as RSSI expectation, but the difference value between the measured value and expected value conforms to the normal distribution. Specifically, suppose $p_{i j}(\mathrm{dBm})$ represents the signal strength value sent from the receiving node $i$ to the sending node $j$, and $p_{i j}$ obeys the Gaussian random variable:

$$
p_{i j} \sim N\left(\bar{p}_{i j}, \sigma_{\mathrm{dB}}^{2}\right)
$$

In Eq. (1), $\bar{p}_{i j}$ is the RSSI expectation of receiving node $i$ to sending node $j$, and $\sigma_{\mathrm{dB}}$ is the standard deviation of the signal attenuation, which reflected the relationship between distance and attenuation of signal strength. Suppose that $d_{i j}=\sqrt{\left(x_{i}-x_{j}\right)^{2}+\left(y_{i}-y_{j}\right)^{2}}$ is the distance between the receiving point $i\left(x_{i}, y_{i}\right)$ and the sending node $j\left(x_{j}, y_{j}\right)$, we can get:

$$
\bar{p}_{i j}=p_{0}-10 n_{\mathrm{p}} \log _{10}\left(\frac{d_{i j}}{d_{0}}\right)
$$

In Eq. (2), $p_{0}$ indicates the received signal intensity $\mathrm{dBm}$ when at reference distance $d_{0}$ and the path loss index is $n_{p}$, in which reference distance $d_{0}$ is usually taken $1 \mathrm{~m}$, and the path loss coefficient $n_{\mathrm{p}}$ is related to the actual environment.

According to Eq. (2), the expectation about receiving signal strength depends on the location $m_{i}\left(x_{i}, y_{i}\right)$ of the receiving node $i$; therefore, the conditional probability that receiving node $i$ has received signal strength $p$ at position $m_{i}$ is:

$$
\begin{aligned}
& p\left(p_{i j=p} \mid m_{i}\right)=\frac{1}{\sqrt{2 \pi \sigma_{\mathrm{dB}}^{2}}} \exp (S), \\
& S=-\frac{\left[p-\left(p_{0}-10 n_{p} \log _{10}\left(\frac{d_{i j}}{d_{0}}\right)\right)\right]^{2}}{2 \sigma_{\mathrm{dB}}^{2}}
\end{aligned}
$$

\subsection{Localization algorithm model based on RSSI-MCL}

The main idea of the MCL positioning algorithm is to estimate the position of the particle in the current moment using the sample particle and its weight. There are three main steps. 


\subsubsection{Stage of location prediction}

Suppose the target node being in a moving state at a certain moment, according to properties of mobile nodes, we can let $m_{k} \sim p\left(m_{k} \mid m_{k-1}\right)$ represent the probability of the node's position that previous time is $m_{k-1}$, and the current time position is $m_{k}$, and this probability distribution is called the transfer distribution.

If the node randomly selects a value from the maximum speed $v_{\max }$ and the minimum speed $v_{\min }$ to be the speed of motion, and randomly selected a value from $[0,2 \pi]$ to be the direction of motion, the transfer distribution $p\left(m_{k} \mid m_{k-1}\right)$ would form a ring with the center of $m_{k-1}$, and the inner radius of $v_{\min }$, the outer radius of $v_{\max }$. Like the following,

$$
p\left(m_{k} \mid m_{k-1}\right)=\frac{1}{\pi v_{\max }^{2}-\pi v_{\min }^{2}}
$$

In the position prediction phase, the position of the previous moment is used to predict the position of the current moment, the possible location of the node obtained from random sampling in the circular area that is described above, and the ring area is the sampling area.

\subsubsection{Stage of filtering}

Filter out the sample that does not meet the conditions according to the location information of the observed value at time $k$, and update the location data of the remaining samples according to the weight. Use $n_{k} \sim p\left(n_{k} \mid m_{k}\right)$ to describe the probability distribution of the measurements of RSSI at a given location, and this probability is the observed distribution.

Specifically, set the location target node to collect a set of samples $\left\{m_{k}^{i}, i=1,2, \mathrm{~L}, \mathrm{~N}\right\}$ from the sampling area where $N$ is the quantity of sample. There is a nonnegative weights $w_{k}^{i}$ in each sample, and the definition is:

$$
w_{k}^{i}=\frac{w_{k}^{* i}}{\sum_{j-1}^{N} w_{k}^{* j}}
$$

In equation, $w_{k}^{i}$ means the weight of samples $i$ at $k$ time. According to the observation distribution, the formula is:

$$
w_{k}^{* i}=w_{k-1}^{* i} p\left(n_{k} \mid m_{k}^{i}\right)
$$

According to the samples and collection $\left\{\left(m_{k}^{i}, w_{k}^{i}\right)\right.$, $i=1,2, \mathrm{~L}, \mathrm{~N}\}$ of corresponding weights, the posterior probability distribution of the node position can be obtained as follows:

$$
p\left(m_{k} \mid n_{1: k}\right)=\sum_{i-1}^{N} w_{k}^{i} \delta\left(m_{k}-m_{k}^{i}\right)
$$

In the equation, $\delta($.$) is the impulse function. After re-$ peated calculation of the node location prediction and weight update, we can obtain the final posterior probability distribution $p\left(m_{k} \mid n_{1: k}\right)$.

\subsubsection{The stage of resampling}

We need to repeat the above prediction and filtering phase to calculate the current position, but after repeated iterations, it is possible that the weight of most samples tends to be zero and only one sample's weight tend to be 1 caused by the problem of algorithm degradation. This degradation means that a large number of calculations are wasted on particles that contributed a few to the posterior probability distribution. In order to avoid this phenomenon, it is necessary to detect the degradation of the algorithm. When the algorithm is degraded, resampling should be performed. When detecting, set effective sample size $N_{\text {eff }}$ and effective sample size threshold $N_{\text {threshold. }}$ Resampling is performed when the effective size is less than the set threshold. The effective sample size is as follows:

$$
N_{\text {eff }}=1 / \sum_{i-1}^{N}\left(w_{k}^{i}\right)^{2}
$$

\subsection{RSSI-IMCL localization algorithm model}

In the prediction phase based on RSSI-MCL algorithm, the position probability distribution of moment $k$ is only related to the position of the moment $k-1$ and the maximum or minimum velocity, and do not taking into account the impact before the moment $k-1$ 's motion on the current node; the motion model of RSSI-MCL algorithm determines node's movement direction according to node's current position. The algorithm considers that the motion in different time periods is independent of each other and may cause nodes to happen some unrealistic activity; in the posterior distribution phase of RSSI-MCL localization algorithm iterative computation position, the process of screening effective particles is slow. Considering the lack of RSSI-MCL localization algorithm above, this paper proposes an RSSI-IMCL.

\subsubsection{Newton interpolation method prediction of motion velocity and moving nodes direction (prediction stage)}

Newton interpolation method is used to estimate the velocity and direction of the current node. Motion prediction is usually based on the smoothness of the node motion trajectory; thus, the localization of the current moment can be estimated by the position of the previous few moments to obtain the movement direction and speed of the current time. Newton interpolation method has the hereditary difference coefficient, as the following:

$$
\begin{aligned}
f\left(x_{0}, x_{1}, x_{2}\right) & =\frac{f\left(x_{1}, x_{2}\right)-f\left(x_{0}, x_{1}\right)}{x_{2}-x_{0}} \\
& =\frac{1}{x_{2}-x_{0}}\left\{\left[\frac{f\left(x_{1}\right)}{x_{1}-x_{2}}+\frac{f\left(x_{2}\right)}{x_{2}-x_{1}}\right]-\left[\frac{f\left(x_{0}\right)}{x_{0}-x_{1}}+\frac{f\left(x_{1}\right)}{x_{1}-x_{0}}\right]\right\} \\
& =\frac{f\left(x_{0}\right)}{\left(x_{0}-x_{1}\right)\left(x_{0}-x_{2}\right)}+\frac{f\left(x_{1}\right)}{\left(x_{1}-x_{0}\right)\left(x_{1}-x_{2}\right)}+\frac{f\left(x_{2}\right)}{\left(x_{2}-x_{0}\right)\left(x_{2}-x_{1}\right)}
\end{aligned}
$$


The divided difference in Newton interpolation method is symmetric. The value of divided difference is not related to the order of the nodes when change the position between the nodes, so it is suitable for the position of nodes in the wireless sensor network.

$$
f\left(x_{0}, x_{1}, \cdots, x_{n}\right)=\sum_{k=0}^{n} \frac{f\left(x_{k}\right)}{\prod_{\substack{j=0 \\ j \neq k}}^{n}\left(x_{k}-x_{j}\right)}
$$

Assuming the position of three moments before are respectively $\left(x_{k-3}, y_{k-3}\right),\left(x_{k-2}, y_{k-2}\right)$, and $\left(x_{k-1}, y_{k-1}\right)$, after the second Newton interpolation of the data of $x$ and $y$, get

$$
\begin{aligned}
x_{k}= & x_{k-3}+3 \times\left(x_{k-2}-x_{k-3}\right)+3 \\
& \times\left(x_{k-1}-2 x_{k-2}+x_{k-3}\right) \\
y_{k}= & y_{k-3}+3 \times\left(y_{k-2}-y_{k-3}\right)+3 \\
& \times\left(y_{k-1}-2 y_{k-2}+y_{k-3}\right)
\end{aligned}
$$

According to the localization of moment $k$, the movement speed and movement direction of the current moment can be calculated as follows:

$$
\begin{aligned}
& \widehat{v}_{t}=\min \left(\sqrt{\left(x_{k}-x_{k-1}\right)^{2}+\left(y_{k}-y_{k-1}\right)^{2}}, v_{\max }\right) \\
& \widehat{\alpha_{k}}=\arctan \left(\frac{y_{k}-y_{k-1}}{x_{k}-x_{k-1}}\right)
\end{aligned}
$$

\subsubsection{Optimize the motion model}

The random waypoint mobility model, RWP, used by the RSSI-MCL localization algorithm does not require high hardware requirements for nodes, but the mobile path is more random. As shown in Fig. $1, n_{k-2}$ and $n_{k-1}$ respectively represent the location of the nodes in the two previous moments. Node $n_{k-1}$ first selects the $n_{k}$ node randomly in the scene as the target location to be reached and move to the target with a random rate $V \in[V \min , V \max ]$. And $\alpha$ is the angle between velocity $v_{k-1}$ and $v_{k}$.The motion of different time periods is independent of each other, which may cause the randomness of nodes' movement direction to be larger and impact locating performance. In Fig. 1, the optimized model of motion, the position prediction equation of moment $k$ is:

$$
\left[\begin{array}{c}
x_{k}^{i} \\
y_{k}^{i}
\end{array}\right]=\left[\begin{array}{c}
x_{k-1}^{i}+v_{k} \cos (\alpha) \Delta k+n_{k} \\
y_{k-1}^{i}+v_{k} \sin (\alpha) \Delta k+n_{k}
\end{array}\right]
$$

In the equation, $\Delta k$ is the environmental impact factor and $n_{k}$ is the Gaussian noise.

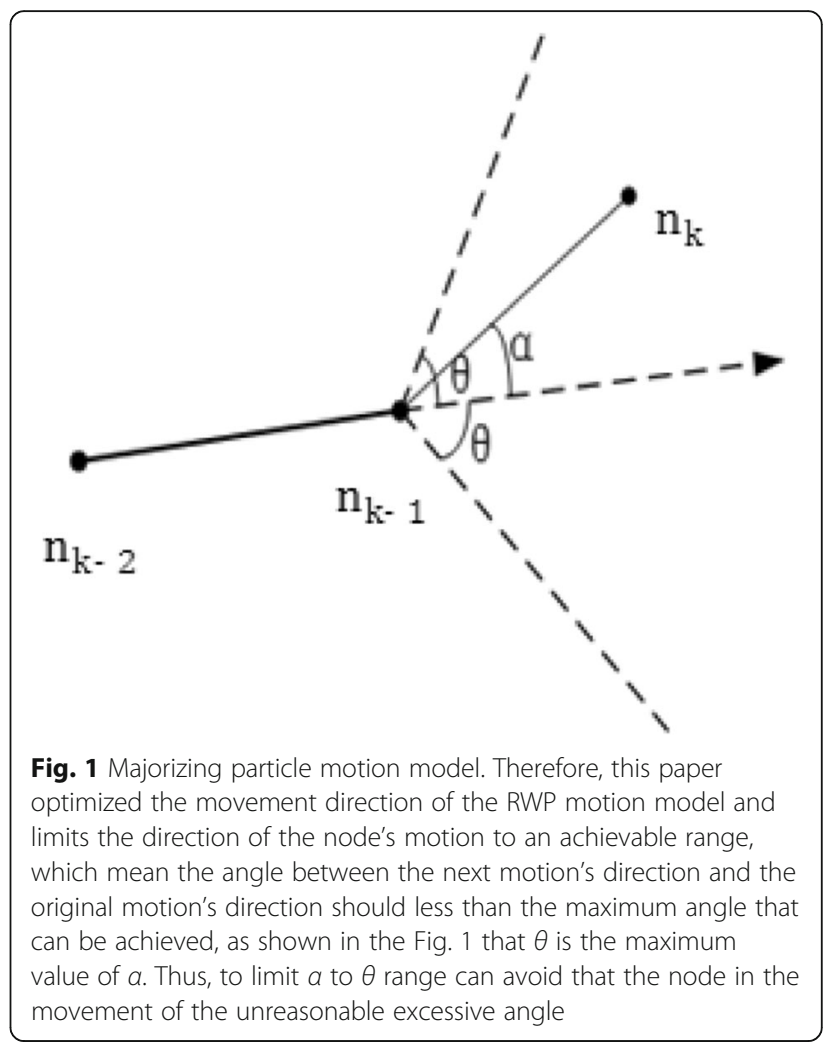

\subsubsection{The particle filter algorithm of importance weight optimization}

Most of predicted particles' weights are all very close to 0 after observation and weighted calculation. We need to do again for location updates and weighted calculations to obtain a particle that has a certain number of weights approaching 1 after multiple iterations. To accelerate the gain of a particle approaching 1 , in this paper, we adopt a particle filter algorithm based on importance weight optimization, introducing an influence factor to optimize the importance weight of the particle, and in the process of resampling, more particles will be duplicated to avoid particle dilution effectively.

In each resampling process, an influence factor $\alpha(0<\alpha<1)$ is added to the weights after normalization, the weight of the particle changing into $\left(\bar{w}_{k}^{i}\right)^{\alpha}$, and then normalized the weight, the value of particle $i$ at moment $k$ is:

$$
\tilde{w}_{k}^{i}=\frac{\left(\bar{w}_{k}^{i}\right)^{\alpha}}{\sum_{i=1}^{N}\left(\bar{w}_{k}^{i}\right)^{\alpha}}
$$

The weight of each particle is retuned, and the weight of the small particles is improved after the optimization of weights, when contrary it gets reduced. The effectiveness of this method is proved by analysis as follows. 
a

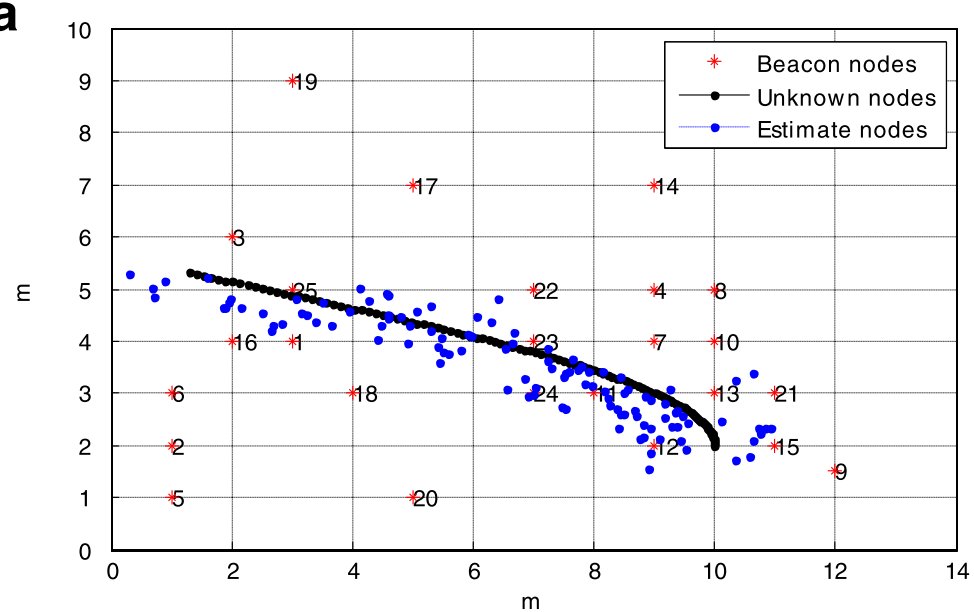

b

MCL

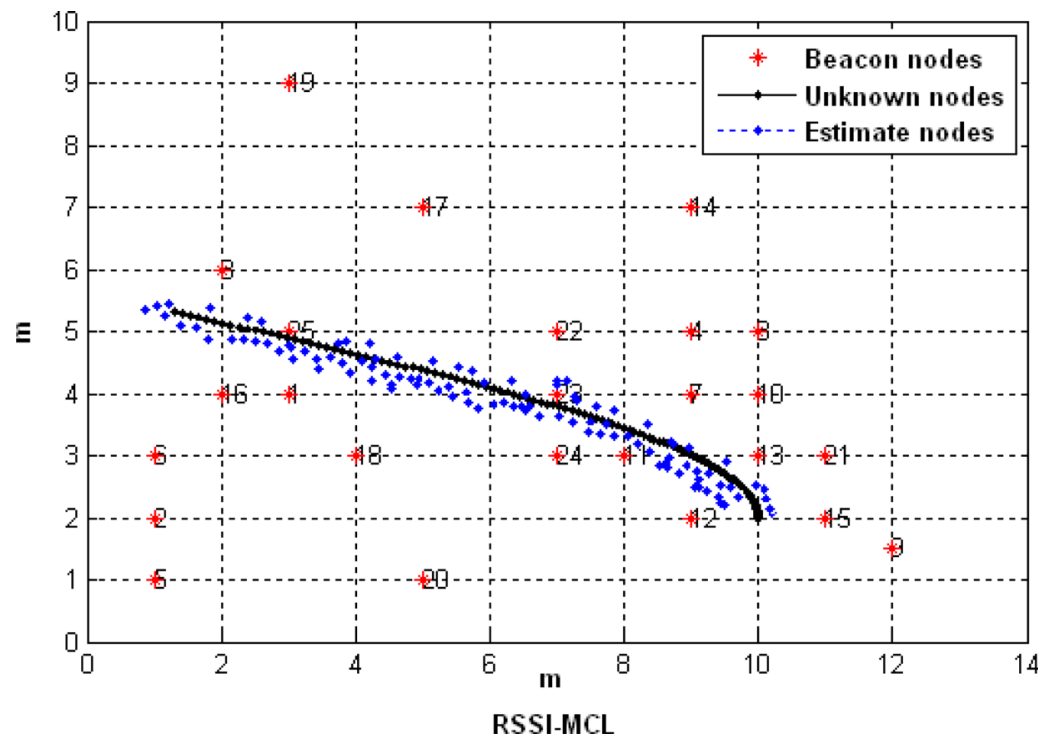

C

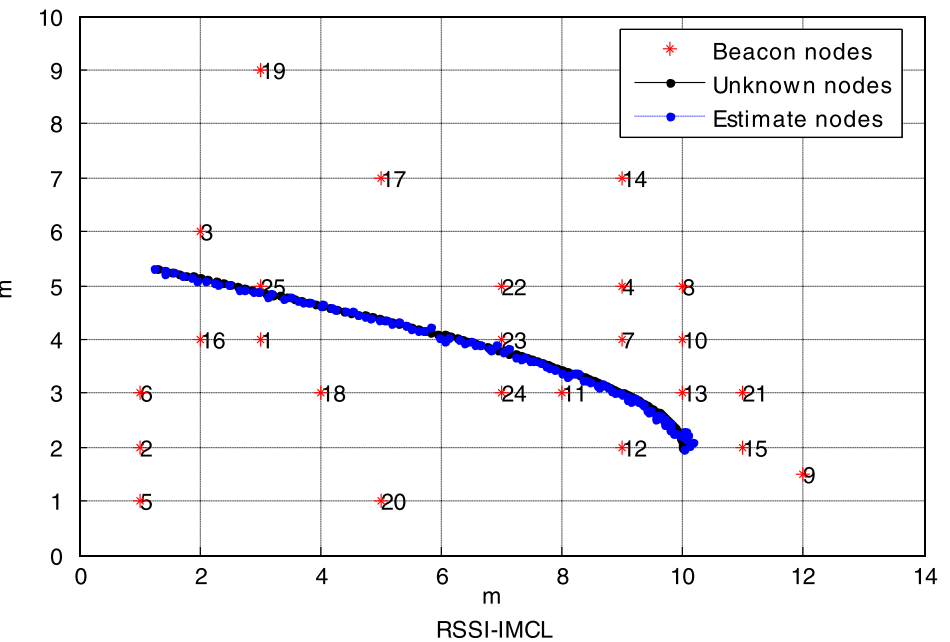

Fig. 2 Network topology. a MCL. b RSSI-MCL. c RSSI-IMCL 
Suppose the particle set that is not normalized in time $k$ is $\left\{x_{k}^{i}, w_{k}^{i}\right\}_{i=1}^{N}$, using (16) to normalize the weight, which is:

$$
\bar{w}_{k}^{i}=\frac{w_{k}^{i}}{\sum_{i=1}^{N} w_{k}^{i}}
$$

Because $0<w_{k}^{i}<1$, it can be assumed that the normalized weight value relationship is $0<w_{k}^{1}<w_{k}^{2}<\ldots$ $<w_{k}^{N}<1$, and the weight after optimization is:

$$
\begin{aligned}
& \tilde{w}_{k}^{i}= \frac{\left(\bar{w}_{k}^{i}\right)^{\alpha}}{\sum_{i=1}^{N}\left(\bar{w}_{k}^{i}\right)^{\alpha}}(0<\alpha<1) \\
&=\bar{w}_{k}^{1} \times \bar{w}_{k}^{2}\left[\frac{1}{\left(\bar{w}_{k}^{1}\right)^{1-\alpha}}-\frac{1}{\left(\bar{w}_{k}^{2}\right)^{1-\alpha}}\right]+\bar{w}_{t}^{1} \times \bar{w}_{t}^{3}\left[\frac{1}{\left(\bar{w}_{k}^{1}\right)^{1-\alpha}}-\frac{1}{\left(\bar{w}_{k}^{3}\right)^{1-\alpha}}\right] \\
& \frac{+\ldots+\bar{w}_{k}^{1} \times \bar{w}_{k}^{N}\left[\frac{1}{\left(\bar{w}_{k}^{1}\right)^{1-\alpha}}-\frac{1}{\left(\bar{w}_{k}^{N}\right)^{1-\alpha}}\right]}{\sum_{i=1}^{N}\left(\bar{w}_{k}^{i}\right)^{\alpha}}
\end{aligned}
$$

As the result of $0<\bar{w}_{k}^{1}<\bar{w}_{k}^{2}<1$ and $(0<\alpha<1)$.

So, $\frac{1}{\bar{w}_{k}^{1}}>\frac{1}{\bar{w}_{k}^{2}}>1 \rightarrow \frac{1}{\left(\bar{w}_{k}^{1}\right)^{1-\alpha}}>\frac{1}{\left(\bar{w}_{k}^{1-\alpha}\right.}>1 \rightarrow \frac{1}{\left(\bar{w}_{k}^{1-\alpha}\right.}-\frac{1}{\left(\bar{w}_{k}^{1-\alpha}\right.}>0$

Similarly, $\quad \frac{1}{\left(\bar{w}_{k}^{1-\alpha}\right)^{1-\alpha}}-\frac{1}{\left(\bar{w}_{k}^{3}\right)^{1-\alpha}}>0, \ldots, \frac{1}{\left(\bar{w}_{k}^{1}\right)^{1-\alpha}}-\frac{1}{\left(\bar{w}_{k}^{N}\right)^{1-\alpha}}>0$

So, $\tilde{w}_{k}^{1}-\bar{w}_{k}^{1}>0$

The small weight of particle will increase when its weight is optimized. And in the process of resampling the particle set $\left\{x_{t}^{i}, w_{k}^{i}\right\}_{i=1}^{N}$, more particles will be copied, avoiding particle degradation effectively.

\section{Comparison of MCL, RSSI-MCL, and RSSI-IMCL}

\subsection{Simulation environment}

This paper's simulation experiment uses MATLAB platform, and the simulation experiment is set in a rectangular plane area of $200 \mathrm{~m} \times 200 \mathrm{~m}$. We assume that 40 beacon nodes are randomly distributed, the position is fixed, and the coordinates are known, and those 80 target nodes were moved randomly, in addition, their direction and speed is random as well, and the movement speed is not exceeding the preset limit. The maximum moving speed of the node is $50 \mathrm{~m} / \mathrm{s}$. Using the RWP model, the communication radius of the beacon node and the target node is both $50 \mathrm{~m}$.

The positioning error used in this paper is defined as follows:

$$
\text { ERROR }=\frac{\sum_{i-1}^{n} \sqrt{\left(x_{i}-x_{i}^{\prime}\right)^{2}+\left(y_{i}-y_{i}^{\prime}\right)^{2}}}{n \times r}
$$

In equation, $n$ is the number of locations, $r$ is the radius of communication, $\left(x_{i}, y_{i}\right)$ is the actual coordinate of the target node, and $\left(\mathrm{x}_{\mathrm{i}}^{\prime}, \mathrm{y}_{\mathrm{i}}^{\prime}\right)$ is the coordinate information estimated by the algorithm.

\subsection{Comparison and analysis of network topology}

What Fig. 2 shows is the topology diagram of MCL localization algorithm, RSSI-MCL positioning algorithm, and RSSI-IMCL localization algorithm. As shown in Fig. 2a, the location of the estimated node is located around the target node. As shown in Fig. 2b, the estimated node location is near the target node, especially in the range of 6-10. As shown in Fig. 2c, the location of nodes estimated by improved RSSI-MCL localization

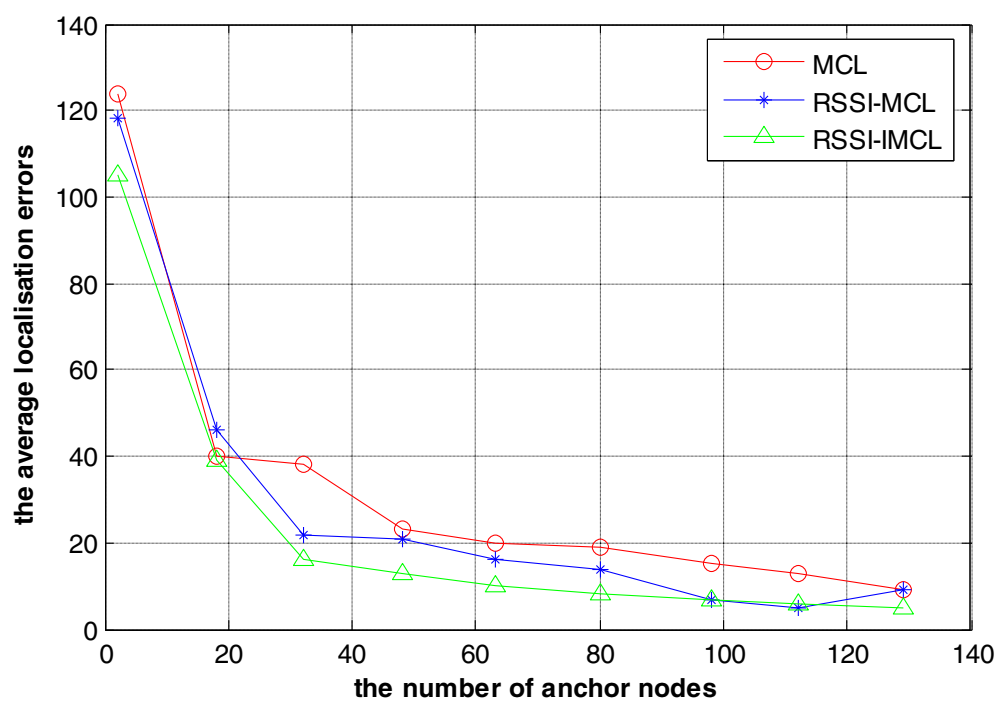

Fig. 3 Comparison of the average localization errors and the number of anchor nodes 


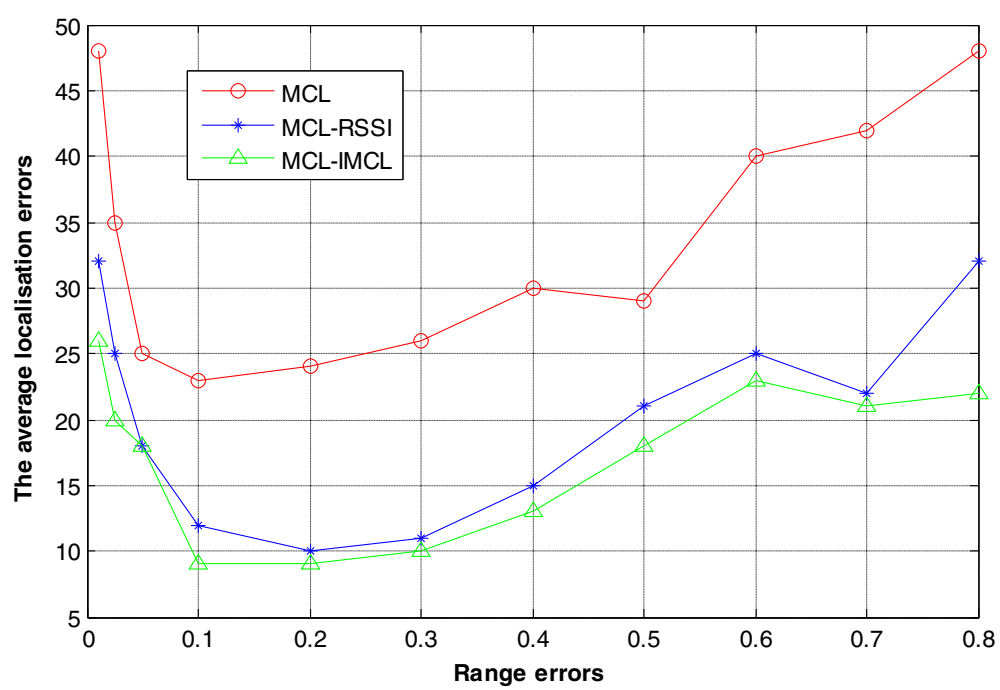

Fig. 4 Comparison of localization errors over different range errors

algorithm is almost identical to the location of the target node, when compared to RSSI-MCL localization algorithm and MCL localization algorithm, it shows better predictive effects.

\subsection{Comparison and analysis of anchor nodes' number and localization errors}

Figure 3 is the variation curve that the average localization errors changed with the number of anchor nodes of MCL, RSSI-MCL, and RSSI-IMCL localization algorithms. With the increase of the number of anchor nodes, the average localization errors of the three algorithms are all decreasing. When the anchor node is within 20, the average localization errors of the three algorithms fluctuate greatly. When the number of anchors is close to 40 , the average localization errors of the three algorithms tend to be stable, and the error of the three algorithms is similar. But the RSSI-IMCL is slightly better than the other two algorithms.

\subsection{Comparison and analysis of localization errors and distance errors}

What the Fig. 4 shows is the curve that localization errors changed with the range error of MCL localization algorithm, RSSI-MCL and RSSI-IMCL. With the increase of ranging error, the average localization errors of the three algorithms are also increasing. However, it can be seen that RSSI-MCL is significantly better than the other two algorithms. When the range errors are between 0.1 and 0.2 , the average localization errors are minimal, then the positioning error gradually increased, so the range errors are optimal when between 0.1 and

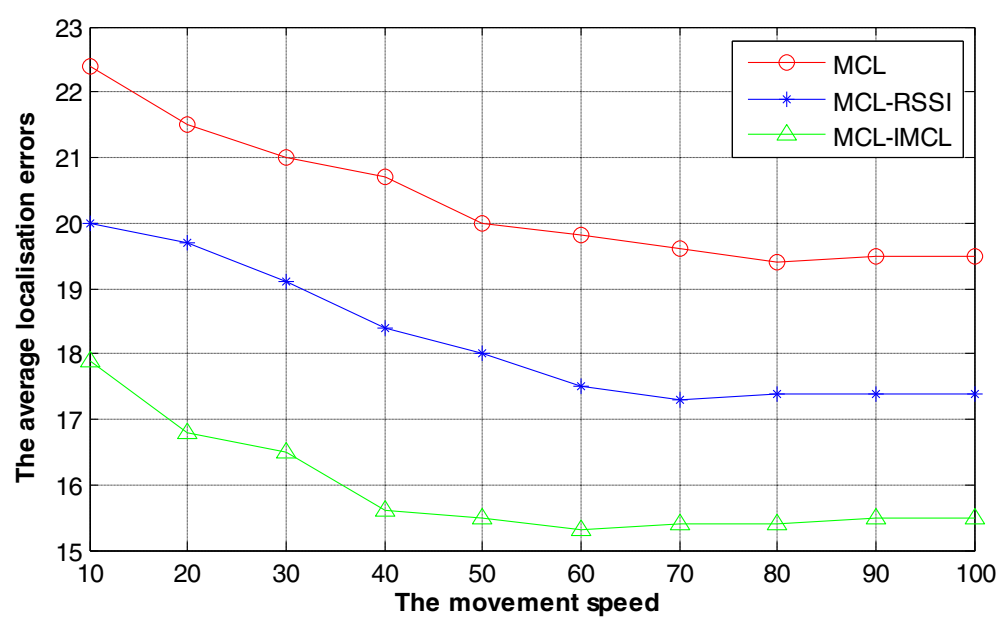

Fig. 5 Comparison of the average localization errors and the movement speed 
0.2 . When the range errors going rewards 0.3 , the average localization errors began to rise.

\subsection{Comparison and analysis of localization errors and movement speed}

What Fig. 5 shows is the curve that the localization errors changed with the particle velocity of MCL, RSSI-MCL, and RSSI-IMCL. The average localization errors of MCL localization algorithm and RSSI-MCL localization algorithm has minimum when the velocity is 20 , then with the movement speed increases, the error also increases. The main reason is that the node speed is increasing and the localization of the next node is increasing so that the non-conforming nodes are difficult to filter. The average localization error of RSSI-IMCL is relatively stable with the increase of velocity, which mainly because of historical trajectory prediction, optimization of particle weights, and the result of the updating particle set.

\section{Conlusion}

In this paper, the mobile node RSSI-IMCL algorithm in wireless sensor networks is proposed, and the random waypoint mobile model is improved. A Newtonian interpolation method is used to estimate the motion speed and direction of moving nodes at the current time. The degradation of particle subset was improved by optimizing weight value. The experimental results show that RSSI-IMCL compares with MCL and RSSI-MCL in different ranging error, anchor node density, and motion speed. RSSI-IMCL algorithm improves network topology and reduces energy loss; the average positioning error was reduced and the positioning accuracy was improved. It is valuable to study the mobile node positioning technology in Wireless Sensor Network. According to the ranging signal, when the distance error is greater, the location error of RSSI-IMCL algorithm will be greater, and how to accurately and quickly cover the network area is the next step. At the same time, the mobile path of the mobile sensor node will also affect the location performance, so the mobile node location algorithm combined with routing and positioning is also the focus of future work.

\section{Abbreviations}

MCB: Monte Carlo Localization Boxed; MCL: Monte Carlo localization; RSSI: Received signal strength indication; RSSI-IMCL: Received signal strength indication improvement Monte Carlo localization; RWP: Random waypoint mobility model; SMCL: Sequential Monte Carlo Localization

\section{Acknowledgements}

The research presented in this paper was supported by Chao Hu University, Hefei, Anhui.

\section{Funding}

The authors acknowledge a key project from the Natural Science Foundation in Anhui Province, China (Grant: KJ2014A096).

\section{Authors' contributions}

$J Y L$ is the main writer of this paper. She proposed the main idea, proposed and deduced the RSSI-IMCL, completed the simulation, and analyzed the result. CW gave some important suggestions for the simulation. Both authors read and approved the final manuscript.

\section{Competing interests}

The authors declare that they have no competing interests.

\section{Publisher's Note}

Springer Nature remains neutral with regard to jurisdictional claims in published maps and institutional affiliations.

\section{Author details}

${ }^{1}$ College of Information Engineering, Chao Hu University, Hefei, Anhui, China. ${ }^{2}$ College of Information Engineering, Anhui Xinhua University, Hefei, Anhui, China.

Received: 19 March 2018 Accepted: 30 May 2018

Published online: 20 June 2018

\section{References}

1. Wang W, Zhu Q. Sequential Monte Carlo localization in mobile sensor networks[J]. Wirel. Netw, 2009, 15(4):481-495.

2. Chelouah L, Semchedine F, Bouallouche-Medjkoune L. A power adjusting anchors with improved localization algorithm for mobile wireless sensor networks[]]. Int. J. Comput. Math. Comput. Syst. Theory, 2017, 1(3-4):129-140.

3. Lei Z. Self-adaptive Monte Carlo localization for mobile robots using range finders[J]. Robotica, 2012, 30(2):229-244.

4. Chelouah L, Semchedine F, Bouallouche-Medjkoune L. A power adjusting anchors with improved localization algorithm for mobile wireless sensor networks[J]. Int J. Comput. Math. Comput. Syst. Theory, 2017, 1(3-4):129-140.

5. Z Liang, X Ma, X Dai, Information-theoretic approaches based on sequential Monte Carlo to collaborative distributed sensors for mobile robot localization. J. Intell. Robot. Syst. 52, 157-174 (2008). https://doi.org/10.1007/ s10846-008-9206-9

6. Shen $X, Y i$ Y, Dong $W$, et al. Mobile nodes localization based on adaptive particle swarm optimization and modified Monte Carlo localization boxed in wireless sensor networks[J]. Sens. Lett., 2014, 12(2):1-12.

7. A Alaybeyoglu, An efficient Monte Carlo-based localization algorithm for mobile wireless sensor networks. Arab. J. Sci. Eng. 40, 1375-1384 (2015). https://doi.org/10.1007/s13369-015-1614-0

8. Kadir H A, Arshad M R. Robust blimps formation using wireless sensor based on received signal strength indication (RSSI) localization method[J]. Sains Malaysiana, 2017, 46(1):129-137.

9. Álvarez Y, M de Cos, J Lorenzo, et al., Novel received signal strength-based indoor location system: development and testing. J Wireless Com Network 2010, 254345 (2010). https://doi.org/10.1155/2010/254345

10. Kumari V R, Nagaraju A, Pareek G. Wormhole attack behaviour in Monte-Carlo localization for mobile sensor networks[J]. J Sensor Technol. 2014, 04(2):48-58.

\section{Submit your manuscript to a SpringerOpen ${ }^{\circ}$ journal and benefit from:}

- Convenient online submission

- Rigorous peer review

- Open access: articles freely available online

- High visibility within the field

Retaining the copyright to your article

Submit your next manuscript at $>$ springeropen.com 